\title{
An Economical Micro-Car Testbed for Validation of Cooperative Control Strategies
}

\author{
Chung H. Hsieh ${ }^{1}$, Yao-Li Chuang ${ }^{2}$, Yuan Huang ${ }^{1}$, Kevin K. Leung ${ }^{1}$ \\ Andrea L. Bertozzi ${ }^{2}$, Emilio Frazzoli ${ }^{3}$
}

\begin{abstract}
This paper describes the design of an economical cooperative control testbed using 1/64 size micro-car vehicles. The vehicles have similar motion constraints to those found in common kinematic models for UAVs. We demonstrate the practical use of this testbed for algorithm validation by implementing a recently proposed UAV-routing algorithm [J. J. Enright et al Proc. AIAA Conf. Guidance, Nav. and Control, 2005]. The experimental results lie within the theoretical bounds predicted for this algorithm.
\end{abstract}

\section{INTRODUCTION}

This paper documents the construction of a small area, cost-effective multiple vehicle testbed. With the widespread study of multiple vehicle coordination on the rise in academia and industry, a vehicle testbed is often necessary to validate the effectiveness of the algorithms. Vehicle testbed are also an invaluable learning tool for students to see theory in action. While many such testbeds exist [1-7], cost per vehicle can be large, on the order of thousands of dollars per vehicle, which can make multi-vehicle studies with more than five vehicles prohibitive. In addition, many of the current testbeds require large area to accommodate a number of vehicles with non-holonomic motion constraints.

Our testbed addresses both issues by using very small and inexpensive radio controlled cars (off-the-shelf cost of approximately $\$ 30$ per vehicle) on a modest size driving surface. We first present all the physical and fiscal dimension of the testbed and its subsystems, including an analysis of measurement errors and a mathematical vehicle model. Second, as a demonstration of the testbed's potential, path planning algorithms are implemented, one for controlling an individual vehicle's dynamics and a multi-vehicle cooperative algorithm.

\section{Multiple Micro Vehicle Testbed}

\section{A. System Overview}

The testbed is comprised of three main systems Fig. 1

1) Vehicles

2) Position Tracking System

3) Control Computer

The vehicles are converted from standard 1/64th scale radiocontrolled cars. The Position Tracking System includes a

\footnotetext{
${ }^{1}$ Dept. of Electrical Engineering, University of California Los Angeles, Los Angeles, CA 90095 chung_hsieh@ieee.org, kevinlgaicedepot.net, yuanhaseas.ucla.edu

${ }^{2}$ Dept. of Mathematics, University of California Los Angeles, Los Angeles, CA 90095 \{chuang, bertozzi\} @math.ucla.edu

${ }^{3}$ Dept. of Mechanical and Aerospace Engineering, University of California Los Angeles, Los Angeles, CA 90095 frazzoli@ucla. edu
}

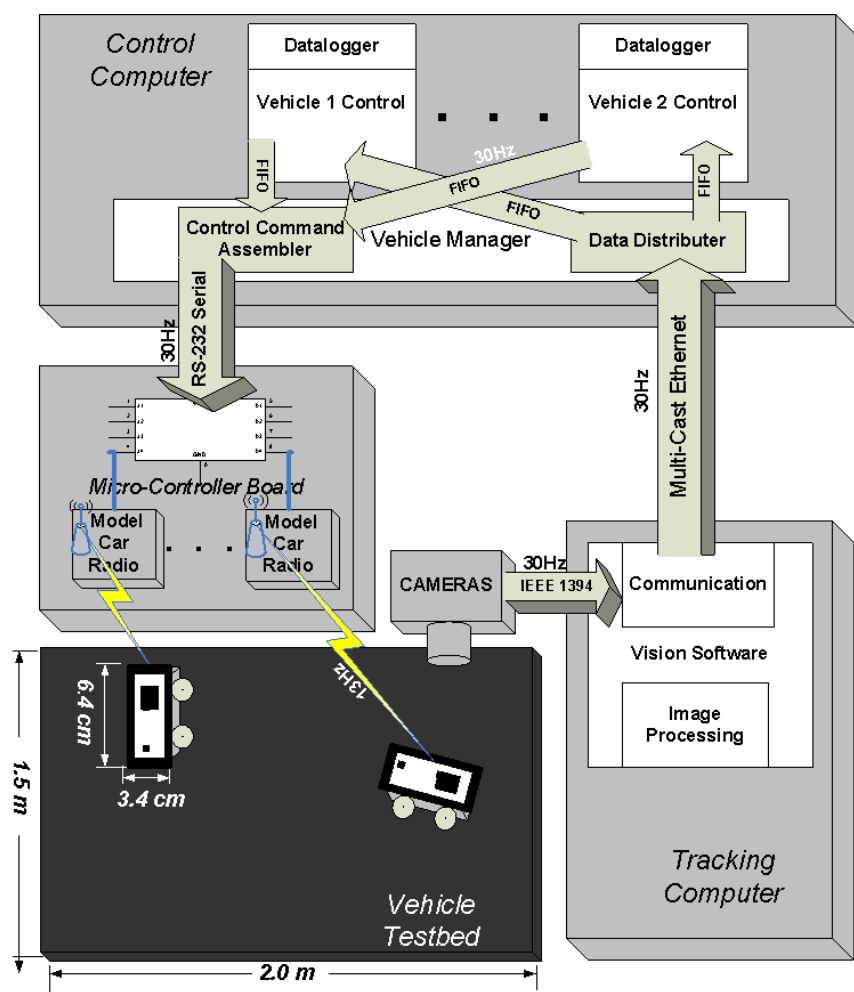

Fig. 1. An system overview of the testbed. All physical components are shown as 3-D objects, software objects are represented in 2-D. The arrows indicate communication connection and direction. The communication rate is shown next to the arrows.

$1.5 m \times 2.0 m$ arena and two monochrome CCD cameras. The vision software is OpenCV for Windows Beta5 [18] with DirectShow Software Development Kit [19] for the camera interface due to camera drivers supplied by the manufacturer The control system is programmed in standard ANSI C, with some POSIX system calls. Despite the mixed platforms, our setup does not introduce additional complexity because the inter-computer communications are conducted via standard networking protocols.

\section{B. Vehicle Information}

We re-engineered off-the-shelf products suitable for the size and scope of the test bed. We chose Hobbico microsizers radio control (RC) cars (1:64) for their low cost, compactness, weight, minimal modification requirements, and ease of purchase. These cars operate on two carrier frequencies $27 \mathrm{MHz}$ and $49 \mathrm{MHz}$ using a Time Division Multiple Access 


\begin{tabular}{|l|c|}
\hline Dimension (L x W X H) & $(6.4 \times 3.4 \times 3.2) \mathrm{cm}$ \\
\hline Weight ( with batteries) & $50 \mathrm{~g}$ \\
\hline Steady State Speed & $60 \mathrm{~cm} / \mathrm{s}$ \\
\hline Acceleration (from zero) & $78 \mathrm{~cm} / \mathrm{s}^{2}$ \\
\hline Turning Radius & $11-13 \mathrm{~cm}$ \\
\hline Angular velocity & $3.3 \mathrm{rad} / \mathrm{s}$ \\
\hline
\end{tabular}

TABLE I

SPECIFICATIONS OF CURRENT TESTBED VEHICLE
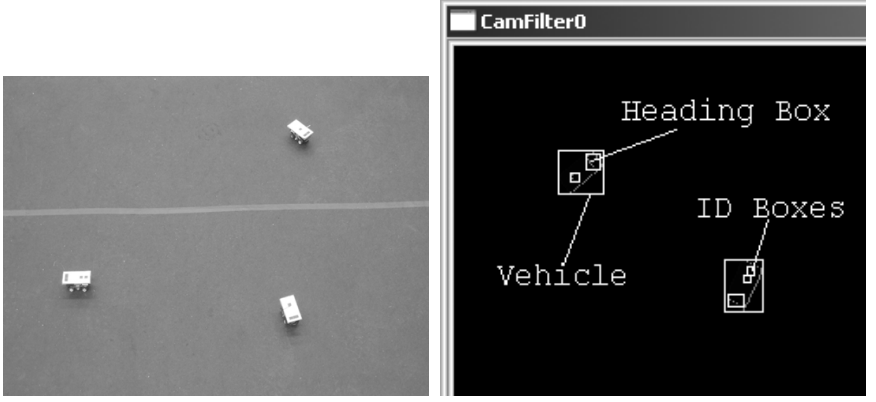

(TDMA) transmission scheme. The commands are updated at $13 \mathrm{~Hz}$. Each channel is capable of controlling three cars simultaneously to allow up to six vehicles. We made slight modifications to both the vehicle and the radio transmitter to match the needs of the test bed. Out of the box, the vehicles have only 5 minutes of continuous run time. To extend the run time to 30 minutes, we replaced the original battery with two AAA batteries secured to the top of the vehicle. We also replaced the transmitter's button switches with electronic switches in order to incorporate this existing radio control architecture into our testbed. However, we could not increase the control update rate which remained at $13 \mathrm{~Hz}$. The vehicle has six states of motion: forward left, forward right, backward left, backward right, forward, and backward. Mechanical limitations apply to both the steering and the velocity control. The steering control is limited to strictly right and left only with a fixed radius. The cars operate with a fixed forward or backward cruise velocity which limits the option of changing the effective velocity to pulsing. Physical characteristics of the vehicle are described in Table I.

\section{Tracking System}

The vehicle arena floor has a top layer of asphalt felt paper, which provides a uniform, non-glossy, black background for imaging. An overhead position tracking system is composed of two Imaging Source DMK 21F04 1/4" Monochrome CCD cameras with progressive scan of 30 images/sec at a resolution of $640 \times 480$ pixels. The cameras are connected via an IEEE 1394a (firewire) interface to a $3.0 \mathrm{GHz}$ PC with IEEE1394 adapter card for image processing. The camera lenses are Pentax H612A(KA) with focal length of $6.0 \mathrm{~mm}$ and $56^{\circ}$ horizontal angle of view. The cameras are mounted 2.6 meters above the testbed resulting in an observable area for each camera of $1.5 m \times 1.1 \mathrm{~m}$, with each pixel representing a $2.4 \mathrm{~mm} \times 2.4 \mathrm{~mm}$ area. The cameras have a $0.144 \mathrm{~m}$ image overlap resulting in a total visible arena of $1.5 \mathrm{~m} \times 2 \mathrm{~m}$. Because the whole vehicle must be visible for tracking, the actual trackable region is slightly smaller, $1.4 \mathrm{~m} \times 1.9 \mathrm{~m}$. The testbed was designed with limited space as a constrain so scalability for us. However, the setup can be expanded easily by adding additional cameras and image processing computers. The testbed was designed with limited space as a constrain so scalability was not an issue for us. However, the setup can be expanded easily by adding more cameras and more image processing computers.

We experienced only minor barrel distortion, common with all camera use. The difference of undistorted radius
Fig. 2. (Left) An unprocessed shot of the testbed. (Right) Partial screen shot of the processed image. The large box surrounds the outer region of the vehicle. The medium size rectangle is the heading box. The small squares denotes the binary identification boxes. In the figure we see car \#2 and car \#3.

from center and distorted radius is 2 pixels resulting in an $0.8 \%$ error on position, which we considered to be small enough to not require a correction in our data. For larger barrel distortions, a correcting filter would be needed before image processing.

The Vehicle Tracking System software utilizes Intel's computer vision software (OpenCV)[18]. We detect the vehicle position and identification using a black and white two dimensional bar code marking similar to that used on the CalTech Multi-Vehicle Wireless Testbed [1,2]. The vehicle information are found with an OpenCV contour searching function and the following algorithm.

1) Record all contrasting shapes detected on the platform and bound them within a rectangle.

2) Sort the rectangles into three groups by size.

3) Assign the large rectangles as possible car object.

4) Go through medium sized rectangles:

- if within bound of a large rectangle, mark as car's heading symbol.

5) Go through small sized rectangles:

- if within bound of a large rectangle, mark as car's ID symbol.

6) Once all rectangles have been sorted and placed, evaluate the car object:

- Position : The center of the large rectangle.

- Heading : the vector formed by the center of the large and medium rectangle.

- Identification : the distance formed by the center of the medium and small rectangles. The distance determines the bit position.

The processed data is then multi-casted via ethernet to the controller computers to close the feedback loop. The whole cycle of identifying and transmitting data for 10 different markers averages $15 \mathrm{~ms}$ per frame.

\section{Control System}

As shown in Fig. 1, the control computer determines the motion of each vehicle. The current testbed architecture has off-board computation, with all n-vehicle's control computed 


\begin{tabular}{|c||c||c|}
\hline & open loop delay $(\mathrm{msec})$ & UAV routing delay $(\mathrm{msec})$ \\
\hline 1 vehicle & 0.7 & 1.5 \\
\hline 2 vehicles & 1.4 & 2.2 \\
\hline 3 vehicles & 2.1 & 2.9 \\
\hline
\end{tabular}

TABLE II

CONTROL SySTEM DELAY

using one computer ${ }^{1}$. However, each vehicle runs an independent process on the computer, and for inter-process communications we used standard named pipes. There is an additional manager program responsible for receiving and distributing the tracking information to each of the $\mathrm{n}$ vehicle programs for control command calculation. Once all vehicle programs generate their respected commands, the manager packages the commands and send it via RS-232 cable to the Micro-Controller Board. The Micro-Controller will unpack the received commands and distribute them to the radio transmitters of the corresponding vehicles. We note that the unavailability of on-board processing prevents us from implementing a truly distributed system. However, when such a system is realized, we no longer need the manager program nor the Micro-Controller Board. The vehicles will directly receive position update from the tracking computer.

Table II shows how the system delay varies with the number of vehicles and algorithm complexity. We measure the delay between when the manager receives new data and issues commands for the two scenarios decribed in section III and IV, respectively. Note that the delay associated with the open loop test can be thought of as a base line, while the UAV routing algorithm bears only modest computational complexity. We have mentioned that the camera captures a frame of image every $33 \mathrm{~ms}$ and the tracking computer requires $15 \mathrm{~ms}$ to process it, leaving the control computer about $18 \mathrm{~ms}$ to compute and issue the commands. Hence there is still a comfortable margin to increase the algorithm complexity and the number of vehicles. Currently, this control system is an Ad hoc design; it will undergo substantial modification once we equip the vehicles with on-board processors (see section V).

\section{BASIC VEHICLE MODEL}

In this section we present a computer simulation model which is helpful for performing virtual experiments for comparison with the testbed. We assume that the vehicle engine has a constant output, the frictional force is linear, and that there is a fixed turning radius for each vehicle. The basic motion model is follows:

$$
\begin{array}{cc}
\dot{x}=v \cos (\theta), & \dot{y}=v \sin (\theta), \\
M \dot{v}=F-\beta v, & \dot{\theta}=\frac{v}{R},
\end{array}
$$

where $x$ and $y$ represent the position coordinates of the vehicle in the laboratory frame, $v$ is the vehicle speed (positive if going forward and negative if going backwards), $\theta$ indicates the angle of the vehicle heading, $M$ is the vehicle

\footnotetext{
${ }^{1}$ The control computer is also a $3.0 \mathrm{GHz} \mathrm{PC}$.
}
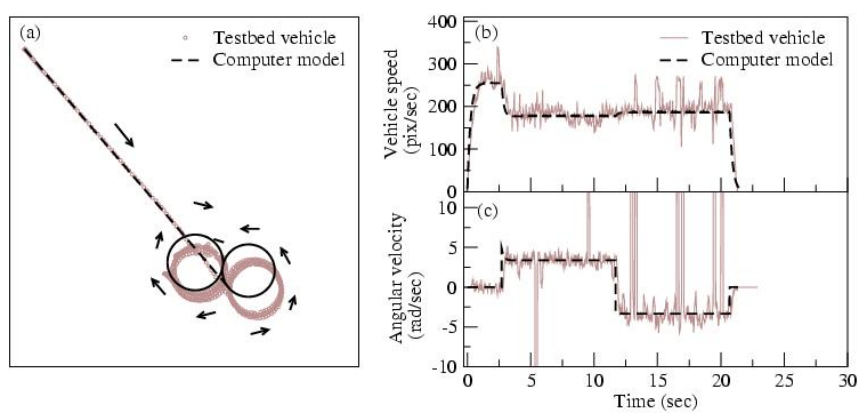

Fig. 3. Comparison of the open loop test to simulation model. (a) The trajectories of the vehicle. (b) Linear speed of the vehicle vs. time. (c) Angular velocity of the vehicle vs. time measured in radians per second. Parameters of the model: $R_{L}=12.61 \mathrm{~cm}, R_{R}=12.73 \mathrm{~cm}, R_{D}=\infty$, $F=62.82 \mathrm{M} \cdot \mathrm{cm} / \mathrm{sec}^{2}, \beta_{L}=1.49 \mathrm{M} / \mathrm{sec}, \beta_{R}=1.42 \mathrm{M} / \mathrm{sec}$, and $\beta_{S}=1.04 \mathrm{M} / \mathrm{sec}$, where $M$ is the vehicle mass. The simulation also takes into account that there is a 0.17 -second response delay of the vehicle to the control signals. Temporal data is filtered by a running 5-point average.

mass, $F$ is the constant driving force of the vehicle, and $\beta$ is the friction coefficient with the floor. The parameter $R$ is the turning radius, with a positive value for a left turn and a negative value for a right turn. For straight forward or backward motion, we set $R=\infty$ so that $\theta$ does not change. The above model is common in the literature on UAV motion planning and is similar to the one studied in [17] but with constant speed.

In practice there are additional factors that affect vehicle motion. The left and right turning radii can be slightly different and varies with vehicle. Second, the vehicle alignment has a slight offset resulting in a slight drift when commanded to go straight. Third, the friction can vary depending on the motion command (left vs. right vs. straight). To address these issues we let $R$ and $\beta$ depend on the direction of turn and also on the particular vehicle in motion.

We compare the model with an open loop test on the testbed. During the test, the vehicle is ordered to go straight, then turn left, and finally turn right. Fig. 3 shows the comparison between the testbed data $\log$ and the computer simulated results. With the model parameters measured from the testbed, both trajectories agree as do the linear speeds and the angular velocities. Our model is second order in linear translation and first order in rotation. However, the equation for $v$ typically has a very short characteristic time. In practice one can simplify the model to first order with a fixed velocity and with very similar results.

\section{A CASE STUdy ON UAV Routing}

As an example of the capabilities of the testbed, we report experimental results from the implementation of a very recent algorithm for UAV routing [16] and compare the observed performance to analytically-derived bounds. Consider a number of vehicles with constant speed and bounded curvature that must visit stochastically-generated targets in a convex, compact two dimensional plane. Targets appear according to a spatio-temporal Poisson process, uniformly in space. We want to minimize the expected waiting time between the appearance of a target and the time it is visited. 
We limit our analysis to the case in which targets appear infrequently.

\section{A. Problem formulation}

Let the environment $\mathcal{Q} \subset \mathbb{R}^{2}$ be a convex, compact set with unit area, and let $\|\cdot\|$ denote the Euclidean norm in $\mathbb{R}^{2}$. Consider $n \geq 1$ vehicles constrained to move at constant speed $v$ along a path with bounded curvature, and let $1 / \rho$ be the maximum curvature. Let the configuration $g_{i} \in S E(2)$ of the $i$-th vehicle $(1 \leq i \leq n)$ be given in coordinates by $g_{i}=\left(x_{i}, y_{i}, \theta_{i}\right)$; the dynamics of each vehicle are given by the ODE (1)-(2).

The vehicles have unlimited range and target-servicing capacity. In the following, we will indicate by $p_{i}=\left(x_{i}, y_{i}\right)$ the position of the $i$-th vehicle. Moreover, we will indicate by $g=\left(g_{1}, g_{2}, \ldots, g_{n}\right) \in S E(2)^{n}$ and $p=\left(p_{1}, p_{2}, \ldots, p_{n}\right) \in$ $\mathcal{Q}^{n}$ the configuration of the $n$-vehicle system, and the positions of the $n$ vehicles, respectively.

Information on outstanding targets - the demand-at time $t$ is summarized as a finite set of target positions $D(t) \subset \mathcal{Q}$, with $m(t):=\operatorname{card}(D(t))$. Targets are generated, and inserted into $D$, according to a homogeneous (i.e., time-invariant) spatio-temporal Poisson process, with time intensity $\lambda>0$, and uniform spatial density. In other words, given a set $\mathcal{S} \subseteq$ $\mathcal{Q}$, the expected number of targets generated in $\mathcal{S}$ within the time interval $\left[t, t^{\prime}\right]$ is

$$
\begin{aligned}
& \mathrm{E}\left[\operatorname{card}\left(D\left(t^{\prime}\right) \cap \mathcal{S}\right)-\operatorname{card}(D(t) \cap \mathcal{S})\right]= \\
& \quad=\lambda\left(t^{\prime}-t\right) \operatorname{Area}(\mathcal{S}) .
\end{aligned}
$$

(Strictly speaking, the above equation holds in the case in which targets are not being removed from the queue $D$.) Servicing of a target $e_{j} \in D$, and its removal from the set $D$, is achieved when the UAV moves to the target's position.

A static feedback control policy for the system is a map $\mu: S E(2)^{n} \times 2^{\mathcal{Q}} \rightarrow\{-v / \rho, 0, v / \rho\}^{n}$, assigning a control input to each vehicle, as a function of the current state of the system, i.e., $\omega_{i}(t)=\mu_{i}(g(t), D(t))$. (The subscript $i$ denotes the component of the output vector, and of the control policy, that are relevant to the $i$-th vehicle.) The policy $\mu$ is stable if, under its action,

$$
m_{\mu}:=\lim _{t \rightarrow+\infty} \mathrm{E}[m(t) \mid \dot{p}=\mu(p, D)]<+\infty,
$$

that is, if the UAV is able to service targets at a rate that ison average - at least as fast as the rate at which new targets are generated.

Let $T_{j}$ be the time that the $j$-th target spends within the set $D$, i.e., the time elapsed from the time $e_{j}$ is generated to the time it is serviced. Define $T_{\pi}:=\lim _{j \rightarrow+\infty} \mathrm{E}\left[T_{j}\right]$ as the steady-state waiting time under the policy $\pi$. Our objective is to minimize the steady-state waiting time, over all stabilizing feedback control policies, i.e.,

$$
T^{*}=\inf _{\mu \text { stable }} T_{\mu}
$$

We now summarize some results from [16], including bounds on the achievable system time $T^{*}$ in light-load conditions, i.e., when $\lambda \rightarrow 0^{+}$, and a routing policy whose performance is provably within a constant factor from the optimal lower bound. In light-load conditions, UAVs which such constrained motion must have holding or loitering pattern while they are waiting for new targets. Optimal loitering patterns depend on the shape of the loitering region; for simplicity we consider circular loitering patterns which allow us to derive algorithms and bounds independent of the shape of the environment.

Define

$$
H_{n}(p, \mathcal{Q})=\int_{\mathcal{Q}} \min _{i}\left\|q-p_{i}\right\| d q
$$

to be the expected distance between $p$ and a point $q$ randomly sampled from a uniform distribution on $\mathcal{Q}$. The function $H_{n}$ is the continuous Weber function or the continuous multimedian function ([14], [13] and references therein), and is equivalent to

$$
H_{n}(p, \mathcal{Q})=\sum_{i=1}^{n} \int_{\mathcal{V}_{i}(p)}\left\|q-p_{i}\right\| d q
$$

where $\mathcal{V}(p)=\left\{\mathcal{V}_{1}(p), \mathcal{V}_{2}(p), \ldots, \mathcal{V}_{n}(p)\right\}$ is the Voronoi partition of the set $\mathcal{Q}$ generated by the points $p$. In other words, $q \in \mathcal{V}_{i}(p)$ if $\left\|q-p_{i}\right\| \leq\left\|q-p_{k}\right\|$, for all $k \in$ $\{1, \ldots, n\}$. The set $\mathcal{V}_{i}$ is referred to as the Voronoi cell of the generator $p_{i}$. The $n$-median of the set $\mathcal{Q}$ is the global minimizer

$$
p_{n}^{*}(\mathcal{Q})=\arg \min _{p \in \mathcal{Q}^{n}} H_{n}(p, \mathcal{Q})
$$

In this paper, we associate to each vehicle a unique and distinct Voronoi cell. Figure 4 shows the Voronoi cells used for our testbed with one, two, and three vehicles. Under the conditions described above, we have [16]

Theorem 1: The system time $T^{*}$ satisfies:

$$
T^{*} \geq \frac{H_{n}^{*}(\mathcal{Q})}{v}+\left(\frac{\pi}{2}-\frac{2}{\pi}\right) \frac{\rho}{v} \approx \frac{H_{n}^{*}(\mathcal{Q})}{v}+0.9342 \frac{\rho}{v} .
$$
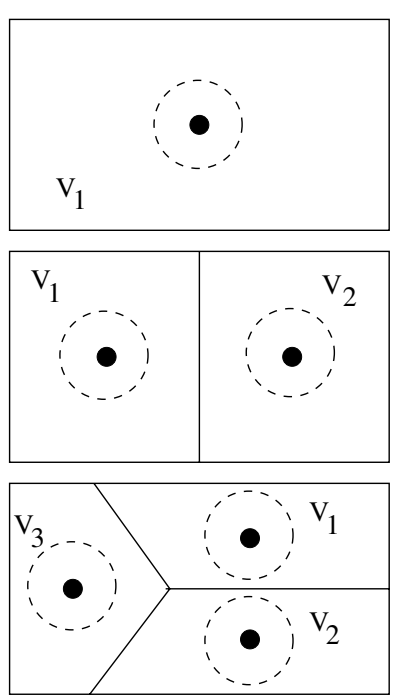

Fig. 4. Loitering regions (Voronoi partition) for the testbed with 1 (top), 2 (middle) and 3 (bottom) vehicles. The dashed lines show the loitering path for each vehicle. 


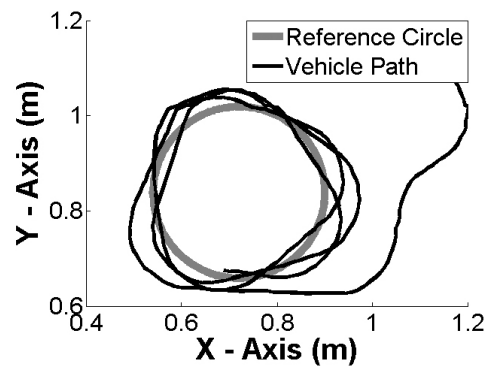

Fig. 5. Measured vehicle path about a reference circle.

Consider the following control policy, which we call the offset median (OM) policy. Let $c^{*}$ be the $n$-median of $\mathcal{Q}$, and define the loitering state for the $i$-th agent as a circular trajectory of radius $\alpha \rho$ centered at $c_{i}^{*}$, with $\alpha \approx 2.918$. In the offset median policy, each agent visits all targets in its own Voronoi region $\mathcal{V}_{i}\left(c^{*}\right)$ in a greedy fashion: in other words, it always pursues the closest target in a Dubins' distance sense. When no targets are available, it returns to its loitering state; the orbit orbit direction chosen to minimize the time to return to the state.

Theorem 2: An upper bound on the system time of the Offset Median policy in light load is

$$
T_{\mathrm{OM}} \leq H_{n}^{*}(Q) / v+\gamma \rho / v \text { as } \lambda \rightarrow 0,
$$

with $\gamma=3.756$.

\section{B. Implementation of $U A V$ routing on testbed}

Fig. 5 shows a sample circle loitering state; it has a $12 \%$ average distance error from the reference circle. Since our vehicles have only three available forward or backward input states (left, right, straight) a hybrid control [9] method makes sense for implementing both the loitering patterns and straight line motion to targets. In our implementation of a point-to-point controller, we deviate from [9] in that when the heading error is within $10^{\circ}$ of the reference angle we use a straight line motion. This less aggressive control law yields a smoother path to the target on our testbed vehicle. We also implement a circular path tracking using the distance error to the desire radius. We select the input that would minimizes the distance error when applied to the vehicle model. We use heuristic and some switching logic to track the circle. By being less aggressive in moving the wheel from side to side, we gain a smoother path along a polygon inside the desired circle. Fig. 5 shows a sample circle loitering state; it has a $12 \%$ average distance error from the reference circle. Figure 6 (top) shows a complete flowchart for the algorithm.

We now present data from the testbed running experiments of the algorithm discussed above. Initially, vehicles are placed at any point and they automatically drive toward their respective loitering state. Due to the size constraint of the testbed, the loitering state for each vehicles are now defined as a circular trajectory of $\alpha \rho$ with $\alpha \approx 1.5$. The corresponding $\gamma$ is 5.54. Once a target appears each vehicles
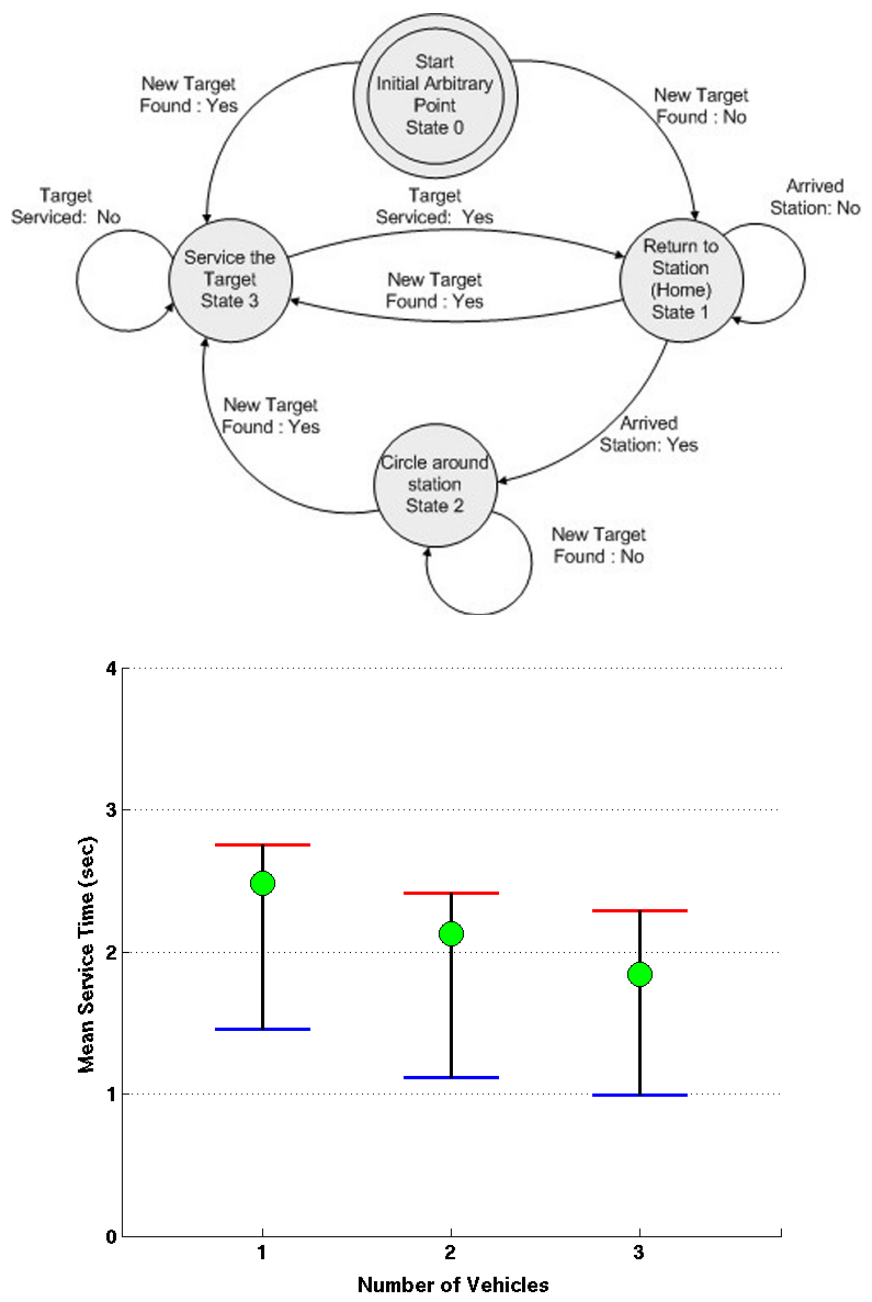

Fig. 6. (top) Vehicle control state diagram. (bottom) Average service time of randomly generated targets in the light load case. Data from three experiments with respectively one vehicle, two vehicles, and three vehicles. The lower and upper bars denote theoretical bounds (3) and (4).

checks whether the target lies within its patrol domain and the vehicle responsible for the target performs a point-topoint maneuver to approach the target. Once the vehicle reaches the target, it returns to its designated loitering state. We generate targets randomly within the testbed arena (using a uniform distribution). In order to guarantee the light load case, targets generated are put into queue only after the vehicle returns to its loitering state. In practice our target generation rate is less than $\frac{1}{6} \mathrm{~Hz}$ and the average service rate is smaller than 6 seconds (see Figure 6). To demonstrate that the performance is within the theoretical upper and lower bounds (resp. (3) and (4), we perform the experiment using 1,2 , and 3 vehicles. In each continuous run, we sequentially generate approximately 200 targets. Less than $10 \%$ of targets generate involve a vehicle approach that either requires more than two passes to reach the target or involves out of bounds motion of the vehicle. We exclude these multi-pass (greater than 2) values from the data collected and compute the arithmetic mean of the service time for the remaining targets. As is expected, the average time decreases as the number of 
vehicles increases and lies within the bounds of the theory described in the previous section. The data is shown in Figure 6. Figure 4 shows the loitering regions and loitering circles used in the experiment.

\section{CONClusions And Future Work}

The aim of this testbed is to address the cost and space constraints typical to many research groups. By using many off-the-shelf items, open-source software and the C programming language we were able to construct a fully operational testbed in twelve weeks with three full time students (two masters students and one undergraduate). Total material cost was under $\$ 4,000$ including the vision and the controls computers. By using small 1/64th scale micro-cars we can increase the vehicle number without requiring a prohibitively large testbed arena. In this paper we experimentally validate recently proposed algorithm for cooperative UAV routing. Our simple testbed vehicles possess the same kind of nonholonomic constraints found in kinematic UAV models, such as finite turning radius and constant speed, and thus serve as a feasible and economical model for testing cooperative control algorithms. Our testbed results, with up to three vehicles running simultaneously, lie within the theoretical bounds for the UAV routing algorithm.

In the future, we plan to build a second generation of vehicles that will have limited on board computing. The new vehicles will have a broader range of motion (variable turn angle and speed) and two-way communication. We plan to use this testbed to study a variety of different cooperative control strategies.

\section{ACKNOWLEDGMENTS}

We thank J. Enright for helpful comments. This research is supported by ARO grant W911NF-05-1-0112, ONR grant N000140410054, NSF grant ACI-0321917, and the Air Force Office of Scientific Research.

\section{REFERENCES}

[1] T. Chung, L. Cremean, W.B. Dunbar, Z. Jin, E. Klavins, D. Moore, A. Tiwari, D. van Gogh, and S. Waydo, A platform for cooperative and coordinated control of multiple vehicles: The Caltech Multi-Vehicle Wireless Testbed, Proc. of the 3rd Conference on Cooperative Control and Optimization, Dec. 2002.

[2] Z. Jin and S. Waydo and E. B. Wildanger and M. Lammers, H. Scholze and P. Foley and D. Held and R. M. Murray, MVWT-II: The Second Generation Caltech Multi-vehicle Wireless Testbed, Proc. of the 2004 American Control Conference, pp. 5321-5326, 2004.

[3] R. DAndrea, Robot soccer: A platform for systems engineering, Computers in Education Journal, 10(1):5761, 2000.

[4] T. W. Mclain and R. W. Beard, Unmanned Air Vehicle Testbed for Cooperative Control Experiments, Proc. of the 2004 American Control Conference, pp. 5327-5331, 2003.

[5] The Minnow Project at Carnegie Mellon University, http://www.cs.cmu.edu/ coral/minnow/.

[6] Robotic Embedded Systems Laboratory, Univ. of Southern California (http://robotics.usc.edu/ embedded/research/videos.html).

[7] A. Stubbs and G. E. Dullerud, Networked control of distributed systems: a testbed, Proc. 2001 ASME International Mechanical Engineering Congress \& Exposition, New York, New York, 2001.

[8] E. Frazzoli and F. Bullo, Decentralized algorithm for vehicle routing in a stochastic time-varying environment, Proc. IEEE Conf. on Decision and Control. Maui, Hawaii. pp. 522-527, 2004.
[9] A. Balluchi, P. Soures and A. Bicchi, Hybrid Feedback Control for Path Tracking by a Bounded-Curvature Vehicle, Proc. 4th International Workshop on Hybrid Systems: Computation and Control. pp. 133-146, 2001.

[10] J. J. Enright and E. Frazzoli. UAV Routing In A Stochastic TimeVarying Enviroment, Proc. International Federation Automation Control, Prague, Czech Republic, July 2005

[11] J. Cortés and S. Martínez and T. Karatas and F. Bullo. Coverage control for mobile sensing networks, IEEE Transactions On Robotics and Automation, 20(2):243-255, 2004.

[12] N. Megiddo and K. J. Supowit. On the complexity of some common geometric location problems, SIAM Journal on Computing, 13(1):182196, 1984

[13] Z. Drezner, editor. Facility Location: A Survey of Applications and Methods. Springer Series in Operations Research. Springer Verlag, New York 1995

[14] P. K. Agarwal and M. Sharir. Efficient algorithms for geometric optimization. ACM Computing Surveys, 30(4):412-458, 1998.

[15] D. J. Bertsimas and G. J. van Ryzin. A Stochastic and Dynamic Vehicle Routing Problem in the Euclidean Plane. Operations Research, 39:601-615, 1991

[16] J.J. Enright and E. Frazzoli and K. Savla and F. Bullo. On Multiple UAV Routing with Stochastic Targets: Performance Bounds and Algorithms. Proc. of the AIAA Conf. on Guidance, Navigation, and Control,August 2005.

[17] L.E. Dubins. On Curves of Minimal Length with a constraint on average curvature and with prescribed initial and terminal positions and tangents. American Journal of Mathematics 79, 497-516, 1957.

[18] http://www.intel.com/technology/computing/opencv/index.htm

[19] http://msdn.microsoft.com/directx 